\title{
Recent Publications of Note ${ }^{1}$
}

\author{
Etzel Cardeña
}

\section{Anomalous Experiences}

1) Corneille J. S., \&, Luke, D (2021). Spontaneous Spiritual Awakenings: Phenomenology, altered states, individual differences, and well-being. Frontiers in Psychology, 12. Doi: 10.3389/fpsyg.2021.720579

Describes and compares Spontaneous Spiritual Awakening with Kundalini Awakening experiences.

2) de Boer, Elpine M. (2020). Out of body, loss of self: Spiritual or scary? Religions $11(558)$. Doi: $10.3390 /$ rell1110558

Distinguishes psychological characteristics related to OBEs being experienced as either positive or distressful.

3) Hirschfeld, T., \& Schmidt, T. T. (2021). Dose-response relationships of psilocybin-induced subjective experiences in humans. Journal of Psychopharmacology, 35(4), 384-397. Doi: 10.1177/0269881121992676

Psilocybin shows a dose-response relation on various indexes of alterations of consciousness.

4) Hurlburt, R. T., Heavey, C. L., Lapping-Carr, L., Krumm, A. E., Moynihan, S. A., Kaneshiro, C., Brouwers, V. P., Turner, D. K., \& Kelsey, J. M. (2021). Measuring the Frequency of Inner-Experience Characteristics. Perspectives on Psychological Science. Doi: $10.1177 / 1745691621990379$

Descriptive experience sampling produces far fewer frequencies of inner-experience phenomena than questionnaires.

\footnotetext{
1 This section contains a list of recent publication which the Editor deems to be of particular interest. Feel free to suggest potential entries for future issues by sending the reference with a link to the paper to etzel.cardena@psy.lu.se
} 
5) Konkoly, K R., Appel, K., Chabani, E., Mangiaruga, A., Gott, J., Mallett, R,... Caughran, B. (2021). Real-time dialogue between experimenters and dreamers during REM sleep. Current Biology 31(7), 1417-1427.e6. Doi: 10.1016/j.cub.2021.01.026

Experimental investigation of real-time communication between researchers and REM sleepers, with 6 of 36 of them providing answers.

6) Luhrmann, T. M., Weisman, K., Aulino, F., Brahinsky, J. D., Dulin, J. C., Dzokoto, V. A., ... Smith, R. E. (2021). Sensing the presence of gods and spirits across cultures and faiths. Proceedings of the National Academy of Sciences of the United States of America, 118(5), e2016649118. https://doi.org/10.1073/pnas.2016649118

The authors propose that absorptive experiences in cultures that see the mind as porous predict the experience of gods and spirits.

See also the exchange about limitations of an instrument/concept used in:

6a) Terhune, D. B., \& Jamieson, G. A. (2021). Hallucinations and the meaning and structure of absorption. Proceedings of the National Academy of Sciences of the United States of America, 118(32), e2108467118. Doi:10.1073/pnas.2108467118 (2021).

6b) Luhrmann, T. M., Weisman, K., Aulino, F., Brahinsky, J. D., Dulin, J. C., Dzokoto, V. A., ... Smith, R. E. (2021). Reply to Terhune and Jamieson: The nature of absorption. Proceedings of the National Academy of Sciences of the United States of America, 118(32), e2109120118. Doi: 10.1073/pnas.2109120118

7) Sierra-Siegert, M., Jay, E.-L., Florez, C. and Garcia, A.E. (2019), Minding the dreamer within: An experimental study on the effects of enhanced dream recall on creative thinking. Journal of Creative Behavior, 53(1), 83-96. Doi:10.1002/jocb.168

Enhancing dream recall increased a measure of creativity, which is related to dissociation and mental thin boundaries. 


\section{Anomalous Cognition}

1) Castagnoli, G. (2021). Unobservable causal loops as a way to explain both the quantum computational speedup and quantum nonlocality. Psychical Review A, 104(032203). Doi: 10.1103/PhysRevA.104.032203

A retrocausal explanation of the quantum computational speedup that may be of relevance to psi phenomena (although the latter are not discussed in the paper itsef).

2) Dean, C., E., Akhtar, S., Gale, T. M., Irvine, K., Wiseman, R., \& Laws, K. R. (2021). Development of the Paranormal and Supernatural Beliefs Scale using classical and modern test theory. BMC Psychology, 9(1):98. Doi: 10.1186/s40359-021-00600-y

A newly developed 13-item scale with good psychometrics.

3) Kruijthoff, D. J., Bendien, E., Doodkorte, C., van der Kooi, C., Glas, G., \& Abma, T. A. (2021). "My body does not fit in your medical textbooks": A physically turbulent life with an unexpected recovery from advanced Parkinson disease After Prayer. Advances in Mind-Body Medicine, 35(2), 4-13.

An anomalous healing event through intercessory patient of a patient with Parkinson's disease.

4) Radin, D., Wahbeh, H., Michel, L., \& Delorme, A. (2021). Psychophysical interactions with a double-slit interference pattern: Exploratory evidence of a causal influence. Physics Essays, 34(1), 79-88. Doi: 10.4006/0836-1398-34.1.79

Radin et al. replicate their previous studies showing a significant effect of selected participants' intention on double-slit interference.

5) Storm, L., \& Tressoldi, P. E. (2020). Meta-analysis of free-response studies 2009-2018: Assessing the noise-reduction model ten years on. Journal of the Society for Psychical Research, 84(4), 193-219.

An MA of recent data continues to find evidence of anomalous communication, particularly using the ganzfeld technique and with selected participants. 
6) Williams, G. R. (2021). Can the psi data help us make progress on the problem of consciousness? Journal of Consciousness Studies 28(5-6), 145-72.

Williams argues that serious consideration of psi research can help clarify the nature of consciousness in general

7) Yang, P., Rhea, P. R., Conway, T., Nookala, S., Hegde, V., Gagea, M.,...Cohen, L. (2020). Human biofield therapy modulates tumor microenvironment and cancer stemness in mouse lung carcinoma. Integrative CancerTherapies. Doi: 10.1177/1534735420940398

Research conducted in a world top cancer research center finds that the experimental condition using a previously-tested psi virtuoso (Sean Harribance) had a significant effect compared with a comparison condition. 